\title{
Dendritic cells as key players in systemic lupus erythematosus
}

\author{
Chamraj Kaewraemruaen, ${ }^{1}$ Patcharee Ritprajak, ${ }^{2,3}$ Nattiya Hirankarn ${ }^{1,4}$
}

\begin{abstract}
System lupus erythematosus (SLE) is a chronic autoimmune disorder affecting multiple organs, and persistent disease activity is associated with increased morbidity and mortality. Impairment of immune cell function and loss of immune tolerance to self-antigens are significant determinants that trigger inflammation and drive SLE pathogenesis. Dendritic cells (DCs) are the most potent antigen-presenting cells that serve as a critical link between innate and adaptive immune system. SLE development and pathogenesis are associated with aberrant regulation in homeostasis and function of DCs, therefore, DC-targeted therapies have become of importance for treatment of SLE and autoimmune diseases. This review focus on the significance of DCs in promoting of SLE pathogenesis, and further discuss the clinical potential of DCs in SLE therapy. The insights on the roles of DCs in SLE will provide the improvement of treatment strategy for SLE patients.
\end{abstract}

Key words: Dendritic cells, Systemic lupus erythematosus, Pathogenesis, Immune tolerance, DC-targeted therapies

\footnotetext{
From:

${ }^{1}$ Center of Excellence in Immunology and Immune-mediated Diseases, Faculty of Medicine, Chulalongkorn University, Bangkok, Thailand

${ }^{2}$ Research Unit in Integrative Immuno-Microbial Biochemistry and Bioresponsive Nanomaterials, Faculty of Dentistry, Chulalongkorn University

${ }^{3}$ Department of Microbiology, Faculty of Dentistry,

Chulalongkorn University, Bangkok, Thailand

${ }^{4}$ Department of Microbiology, Faculty of Medicine,

Chulalongkorn University, Bangkok, Thailand
}

\section{Introduction}

Systemic lupus erythematosus (SLE) is a chronic autoimmune disorder characterized by autoantibody production, immune complex deposition, and multi-organ involvement. The etiology of SLE still remains elusive; however, multiple factors, including genetic susceptibility, age and hormonal factors, and environmental risk, have been suggested to influence disease onset and pathogenesis. Underlying mechanisms of SLE pathogenesis, involving in dysregulation of innate immune cells, loss of B- and T-cell tolerance to self-antigens, and perpetuate cytokine production, propagate inflammation and tissue damages.

Dendritic cells (DCs) are innate immune cells acting as antigen-presenting cells (APCs) that central to immune activation and immune tolerance. Types of stimuli and environmental factors are critical for facilitating DCs to develop their multifaceted functionality that determines the outcome of adaptive immune response. Hyperresponsiveness and altered tolerogenicity of DCs are associated with autoimmune disease development and pathogenesis, therefore, DC-targeted therapies aiming at induction of self-tolerance have become

\section{Corresponding author:}

Patcharee Ritprajak

Research Unit in Integrative Immuno-Microbial Biochemistry and Bioresponsive Nanomaterials, Department of Microbiology, Faculty of Dentistry, Chulalongkorn University

34 Henri-Dunant Road, Pathumwan, Bangkok, 10330, Thailand

E-mail: Patcharee.R@chula.ac.th

a promising approach for autoimmune disease treatment. Mounting evidences have described pathogenic roles of DCs in SLE, and pointed toward the significance of DCs in SLE therapy. Herein the implication of DCs in SLE pathogenesis and the clinical potential of DC-targeted therapies in SLE are discussed.

\section{Dendritic cell subsets}

Dendritic cells are heterogeneous cell population, which commonly classified into two lineages; conventional DCs (cDCs) and plasmacytoid DCs (pDCs), which differ in phenotypic and functional properties. The cDCs confer superior to $\mathrm{T}$ cell activation and differentiation through their abilities of antigen presentation, co-signal transmission and cytokine production. In contrast, $\mathrm{pDC}$ are poor in $\mathrm{T}$ cell activation due to their low expression level of MHC class II and co-stimulatory molecules. Conventional DCs express the transcription factor, zinc finger and BTB domain containing 46 (Zbtb46), and are further categorized into two subtypes, $\mathrm{CDC} 1$ and $\mathrm{CDC} 2$ based on their lineage transcription factors, 
phenotype and function. Two major transcription factors, basic leucine zipper transcriptional factor ATF-like 3 (BATF3) and interferon regulatory factor (IRF) 8 are required for cDC1 development, while IRF4 is critical for cDC2 commitment. Plasmacytoid DCs express the specific transcription factor, E-protein transcription factor 4 (TCF4 or E2-2) that regulates their development and function., ${ }^{1,2}$ In this review, we exclude epidermal Langerhans cells (LCs) because several recent evidences have unveiled that the primitive origins of LCs are yolk sac macrophages and fetal liver monocytes, and phenotype and function of LCs are in between DCs and macrophages. ${ }^{3,4}$

\section{Murine DCs}

Mouse model is commonly used for preclinical study to assess therapeutic implication of DCs so it is important to understand the subset of DC in mice as well. Murine cDCs residing in lymphoid tissues (lymph nodes and spleen) and non-lymphoid tissues are called lymphoid tissue-resident cDCs and migratory cDCs, respectively. The common markers of murine $\mathrm{cDCs}$ are $\mathrm{CD} 11 \mathrm{c}$ and MHC class II, and the distinct $\mathrm{cDC}$ subtypes are further identified by additional markers. CD8a is uniquely expressed on lymphoid tissue-resident $\mathrm{CDC} 1$ while CD103 is the major marker of migratory cDCs. Both CD8a resident DCs and CD103 migratory DCs share the phenotypic markers, C-type lectin receptor (Clec) 9A (also known as dendritic cell NK lectin group receptor, DNGR1), XCR1 and CD24. ${ }^{1} \mathrm{cDC} 1$ s produce IL-12 and IFN- $\gamma$ which orchestrate $\mathrm{T}$ helper (Th) 1 differentiation, and they are particularly capable of cross-presentation to induce cytotoxic $\mathrm{T}$ lymphocyte (CTL) response. ${ }^{5,6}$ Besides, cDC1s play a role in the maintenance of peripheral tolerance by secreting TGF- $\beta$ to mediate antigen-specific FoxP3 ${ }^{+}$regulatory $\mathrm{T}$ cells (Tregs). ${ }^{7,8} \mathrm{cDC} 1 \mathrm{~s}$ have been shown to express high level of indoleamine 2,3-dioxygenase expression (IDO) and they have become more tolerogenic upon IFN stimulation in a IDO-dependent manner. ${ }^{9}$ Murine intestinal CD103 ${ }^{+}$DCs also express IDO and this subset play a crucial role in the maintenance of mucosal tolerance. ${ }^{10}$

The phenotypic markers of murine lymphoid tissue-resident cDC2s is CD8 $a^{-} \mathrm{CD}_{11} \mathrm{~b}^{+}$dendritic cell immunorecptor $2(\mathrm{DCIR} 2)^{+}$signal regulatory protein $\alpha(\mathrm{SIRP} \alpha)^{+}$, while the

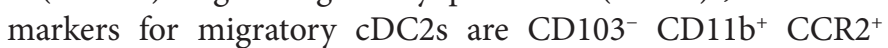
$\mathrm{SIRPa}^{+} \mathrm{CX} 3 \mathrm{CR} 1^{+}$. $\mathrm{cDC} 2 \mathrm{~s}$ preferentially mediate Th2 and Th17 response under the regulation of IRF4. ${ }^{11,12}$ Furthermore, lymphoid tissue-resident cDC2s could support humoral immunity by promoting $\mathrm{T}$ follicular helper (Tfh) differentiation through Notch-2-dependent mechanism. ${ }^{13}$ cDC2s also participated in immune tolerance by triggering Treg differentiation in thymus and peripheral tissues. ${ }^{14,15}$ Murine cDC2s expressed low level of IDO, however, they could be tolerogenic in response to IFN- $\gamma$ but independent on IDO activity. ${ }^{16}$

Plasmacytoid DCs are specialized cells that potentially produce high level of type I IFN, especially in a Toll-like receptor (TLR) 7 and TLR9 dependent fashion. Murine pDCs are characterized as $\mathrm{CD} 11 \mathrm{c}^{\mathrm{lo}} \mathrm{MHC}$ class $\mathrm{II}^{\mathrm{lo}} \mathrm{B} 220^{+}$Siglec $-\mathrm{H}^{+}$ and BM stromal cell Ag-2 (BST2; CD137; plasmacytoid dendritic cell antigen-1 or PDCA- 1$)^{+}$cells. ${ }^{1}$ In steady state, pDCs are innate inducers that maintain peripheral tolerance and prevent inflammation. A recent study has demonstrated that pDCs employed IDO activity to promote $\mathrm{CD}^{+} \mathrm{Fox}-$ $\mathrm{p}^{3}{ }^{+}$Treg cell differentiation and protect against atherosclerosis. ${ }^{17}$

\section{Human DCs}

The investigation and characterization of human DC lineage are limited due to the dilemma of the in vivo study. At present, human myeloid and lymphoid tissue-resident DCs are better characterized, while the migratory DCs are unclearly identified. Human cDC1s are characterized by CD141 (blood dendritic cells antigen (BDCA) 3) expression, while cDC2s are restricted to CD1c (BDCA1) expression. ${ }^{2}$ Human and murine $\mathrm{cDCs}$ share the transcriptional signatures and phenotypes, but differ in some functional features. Both human $\mathrm{cDC} 1 \mathrm{~s}$ and $\mathrm{cDC} 2 \mathrm{~s}$ could produce IL-12, and induce the subsequent Th1 immunity, and activation of these cDCs by thymic stromal lymphopoietin (TSLP) skewed $\mathrm{T}$ cells toward Th2 response in allergic diseases. ${ }^{18,19}$ Only a couple studies have demonstrated the implication of human cDCs in immune tolerance. The blood circulating $\mathrm{CD} 141^{+} \mathrm{CD} 163^{+}$ cDC1s producing IL-10 were found to potentially induce IL-10 producing Tregs, while CD1c ${ }^{+}$cDC2s secreted IL-10, IL-27 and TGF- $\beta$ in response to infection to inhibit effector $\mathrm{T}$ cells and expand IL-10 producing Tregs..$^{20,21}$

The lineage markers for human pDCs are CD45RA, CD123, CD303 (BDCA2) and CD304 (BDCA4). ${ }^{2}$ Similar to murine pDCs, substantial type I IFN production of human pDCs is obligated to TLR7 and TLR9 activation. Similar to murine pDCs, human pDCs also participate in the maintenance of immune tolerance. Human pDCs exploited IDO, granzyme B, and inducible T-cell co-stimulator ligand (ICOSL) to mediate Treg expansion and regulate peripheral tolerance. ${ }^{17,22,23}$

\section{Role of dendritic cells in systemic lupus erythe- matosus}

Although, the mechanism how the immune tolerance is disrupted in SLE is not yet well clarified, DCs are thought to play a key role in initiation, amplification and perpetuation of disease (Figure 1). Several studies have reported the significant alterations in frequency, phenotypes and functions of DCs in SLE patients when compared to those of healthy individuals. For example, the decreased frequencies of blood circulating $\mathrm{cDCs}$ and pDCs with no perturbation of their functions were related to disease activity in patients with active SLE, while the increased accumulation of these DCs in the affected tissues was associated to the tissue inflammation and damages. ${ }^{24,25}$ Similar to human, lupus prone mice showed high infiltration of pDCs and $\mathrm{cDCs}$ in spleens and kidneys at the progressive period of disease. ${ }^{26,27}$ These data suggest that DC accumulation in the organs may contribute to the tissue inflammation and SLE pathogenesis.

The increased apoptotic cells as a result of dysregulated cellular apoptosis and defective clearance of dying cells are perhaps initiators of tissue injuries and SLE pathogenesis. The high levels of apoptotic cells were detected in serum, lymphoid organs and inflamed tissues in patient with SLE. ${ }^{28}$ Self-RNA and self-DNA released from apoptotic cells are the 


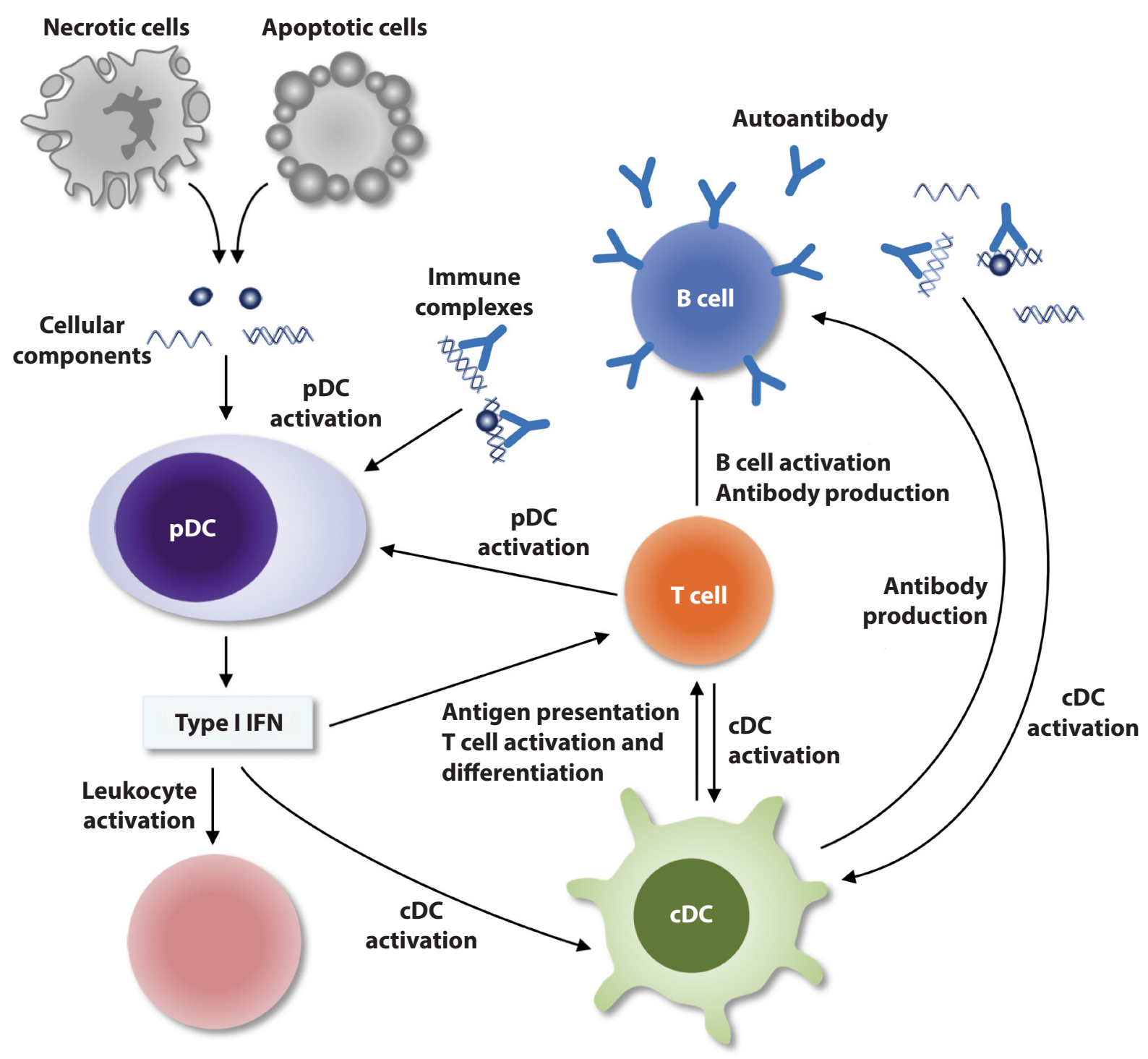

Figure 1. Potential roles of DCs in promoting SLE pathogenesis.

In SLE, clearance of dying cells is defective which lead to persistent releasing cellular components that vigorously stimulate DCs. Activated pDCs secrete type I IFN to promote innate and adaptive immune responses, while activated cDCs present self-antigens and stimulate autoreactive lymphocytes. Effector lymphocytes in turn enhance the capacity and ability of DCs, resulting in amplification and perpetuation of inflammation and SLE pathogenesis.

source of stimuli which activate pDCs via the engagement of TLR7 and TLR9 and retinoic acid-inducible gene 1 (RIG-I)like receptors (RLRs), and consequently result in robust type I IFN production. ${ }^{29,30}$ The levels of type I IFN were markedly elevated in the serum of SLE patients, and the spleens of lupus prone mice were correlated to the increased number of activated pDCs. ${ }^{27,31}$ The deficient in type I IFN in murine lupus model showed the reduction in anti-DNA antibodies and glomerulonephritis pathology. ${ }^{32}$ In parallel, deletion of pDCs in lupus prone mice before disease initiation resulted in the impaired autoreactive $\mathrm{T}$ and $\mathrm{B}$ cells, reduced autoantibody production, decreased type I IFN expression, and ameliorated organs pathogenesis. ${ }^{33}$ Type I IFN was also involved in the activation of monocytes, neutrophils, and adaptive immune cells as well as further induces cDC differentiation. ${ }^{34,35}$ It has been well-known that type I IFN plays a critical role in conversion of Th1, however, the role of type I IFN in Th17 induction is still ambiguous. Evidences have demonstrated that TLR7-activated pDCs promoted autoimmune Th17 response, and high levels of type I IFN were related to high numbers of Th17 in serum of SLE patients. ${ }^{36,37}$

Other stimuli influencing pDC stimulation and type I IFN production have also been described. High mobility group box 1 protein (HMGB1) is a ubiquitous nuclear protein passively released by necrotic cells, which was identified as a danger-associated molecular patterns (DAMPs) that can trigger inflammation and activate immune cells. HMGB1 binds to TLRs and receptor for advanced glycation end products (RAGE) and transduces signal through nuclear factor kappa light chain enhancer of activated $B$ cells $(N F-\kappa B)$ pathway. ${ }^{38}$ High levels of HMGB1 were presented in serum of SLE patients, and correlated to the levels of serum type I IFN. ${ }^{39}$ HMGB1 in complex with DNA facilitated the activation of TLR9 and RAGE on pDCs and resulted in robust type I 
IFN production. ${ }^{40}$ Elevated circulating immune complexes in serum of SLE patients have been shown to be correlated with disease severity. ${ }^{41}$ Nucleic acid-containing immune complexes were internalized by pDCs via Fc $\gamma$ RIIa into endosome where they activated TLR7 and TLR9 and resulted in type I IFN production. ${ }^{42}$ In addition, type I IFN production by IC-stimulated pDCs were markedly enhanced in the presence of activated T cells. ${ }^{43}$

Conventional DCs also play an important role in SLE onset and pathogenesis. The in vitro studies using human monocyte-derived DCs (MoDCs) and murine bone marrow-derived DCs (BMDCs) allow the understanding of the roles of cDCs in SLE. Apoptotic cells elicited MoDCs to undergo maturation and produce high level of IL-6, a key cytokine for Th17 polarization. ${ }^{44}$ In line, apoptotic cell-activated BMDCs showed the increased capacity to induce Th17 response. ${ }^{45}$ The enhanced IgG production by plasmablasts also affected cDC differentiation and function in SLE. The interaction between IgG-containing immune complexes and TNF receptor I (TNFR1) on monocytes was required for cDC differentiation. The immune complexes also promoted Fc $\gamma \mathrm{R}$-mediated endocytosis in cDCs, which conferred the amplified immune response. ${ }^{46,47}$ The in vivo study demonstrated that adoptive transfers of splenic DCs from aged lupus mice could induce B cell hyperactivation, exacerbate autoantibody production, and enhance DC and Th1 differentiation and expansion in pre-autoimmune young mice. ${ }^{48}$ On the contrary, a recent study has demonstrated that $\mathrm{CDCs}$ were not required for autorective $\mathrm{B}$ cell activation, and cDC-specific ablation markedly enhanced extrafollicular (EF) plasmablast response to self-antigens, which the underlying mechanism may be via the regulation of Tfh responses. ${ }^{49}$

Deficit in DC immunoregulatory function is also observed in SLE patients. The spontaneous overexpression of a co-stimulatory molecule, CD86, was found in DCs derived from monocytes of SLE patients, and these DCs hyper-responded to the activation signal, leading to the high production of IL$6 .^{50}$ Circulating pDCs and cDCs from SLE patients displayed the downmodulated expression of the inhibitory molecules, immunoglobulin-like transcript (ILT) 3 and ILT4, which are crucial for tolerogenicity of DCs. ${ }^{51,52}$ Leukocyte-associated immunoglobulin-like receptor 1 (LAIR-1 or CD33) is a receptor of complement C1q, and their interaction inhibits DC differentiation and activation. The expression of LAIR-1 was significantly reduced in pDCs of patients with juvenile SLE, suggesting the loss of $\mathrm{pDC}$ immunoregulatory properties. ${ }^{53}$

\section{Therapeutic potential of dendritic cells in system- ic lupus erythematosus}

DCs have become a therapeutic target for SLE and other autoimmune diseases because they participate in the immune dysregulation that contributes to the initiation and perpetuation of SLE pathogenesis. Although, anti-inflammatory and immunosuppressive medications have been widely used for SLE treatment, these therapies are non-specific and elicit clinical adverse effects. Therefore, therapeutic strategies that restore immune tolerance to self-antigens are turning into promising strategies because they are more specific and lesser side effects. Since DCs can be manipulated to restore Tand B-cell tolerance in an antigen-specific manner, a clinical manipulation of DCs to either decrease their immunogenicity or increase their tolerogenicity have been of high interest for autoimmune disease treatment.

Tolerogenic DCs (tol-DCs) are DCs with immunosuppressive properties and abilities to induce immune tolerance. The phenotypic and functional features of tol-DCs depend on extrinsic and intrinsic factors, and the state of maturation. Ex vivo tol-DCs are generated from peripheral blood monocytes, bone marrow, and cord blood progenitor cells and are subsequently conditioned with pharmacologic interventions to induce tolerogenicity. The common phenotypes of tol-DCs are reduced expression of $\mathrm{T}$ cell co-signaling and MHC molecules, upregulated expression of immuomodulatory molecules, decreased production of pro-inflammatory cytokines, enhanced production of immunosuppressive cytokines, and high capacity of Treg induction (Figure 2). TolDCs can be induced by a wide variety of pharmacologic interventions, including immunosuppressive drugs, cyclic AMP inducers, chemicals, proteins and neuropeptides and cytokines. ${ }^{54}$ However, tolerogenicity of DCs is varied among the drugs and protocols, and it should be concerned for the treatment outcome of autoimmune diseases..$^{55}$

To date, autologous tolDC-based therapy is ongoing clinical trials for the treatment of type 1 diabetes mellitus (T1D), rheumatoid arthritis (RA), multiple sclerosis (MS) and Crohn's disease. Tolerogenic DC-based therapy is promising so far for SLE patients as well. A few evidences have explored tolDC induction from autologous monocytes of SLE patients. Initially, apoptotic cells opsonized with iC3b was capable of induction of tol-DCs with low maturation and resistant to inflammatory stimuli, however, these apoptotic cells failed to generate tol-DCs from monocytes of SLE patients. ${ }^{56}$ Thereafter, treatment of MoDCs from SLE patients with the combination of 1,25-dihydroxyvitamin D3 (VitD3) and dexamethasone could successfully generate tol-DCs with the capability of high IL-10 production, induction of IL-10 producing Tregs, and suppression of IFN- $\gamma$ and IL-17 producing $\mathrm{T}$ cells. ${ }^{57} \mathrm{~A}$ recent study has demonstrated that culture of MoDCs from SLE subjects in the presence of dexamethasone together with rosiglitazone could skew DCs toward tolerogenic phenotypes, and these DCs exhibited the reduced expression of co-stimulatory molecules, decreased IL-6 and IL-12 production, and high IL-10 production in response to lipopolysaccharide (LPS). In addition, the generated tol-DCs were resistant to apoptotic cells-induce maturation, and suppressed allogeneic $\mathrm{T}$ cells proliferation in vitro. ${ }^{58}$ Intriguingly, the tolerogenic probiotics, Lactobacillus delbrueckii and $L$. rhamnosus were capable of inducing tol-DCs from MoDCs of SLE patients, and the tolerogenic probiotics-treated DCs displayed downmodulate expression of maturation markers. Furthermore, the expression of IDO and IL-10 was significantly unregulated while IL-12 was decreased in the tolerogenic probiotics-treated DCs. ${ }^{59}$ Although safety and feasibility of autologous tol-DCs generated from monocytes of SLE patients should be further investigated, these data show the exciting prospect of autologous tol-DCs in SLE therapy. 

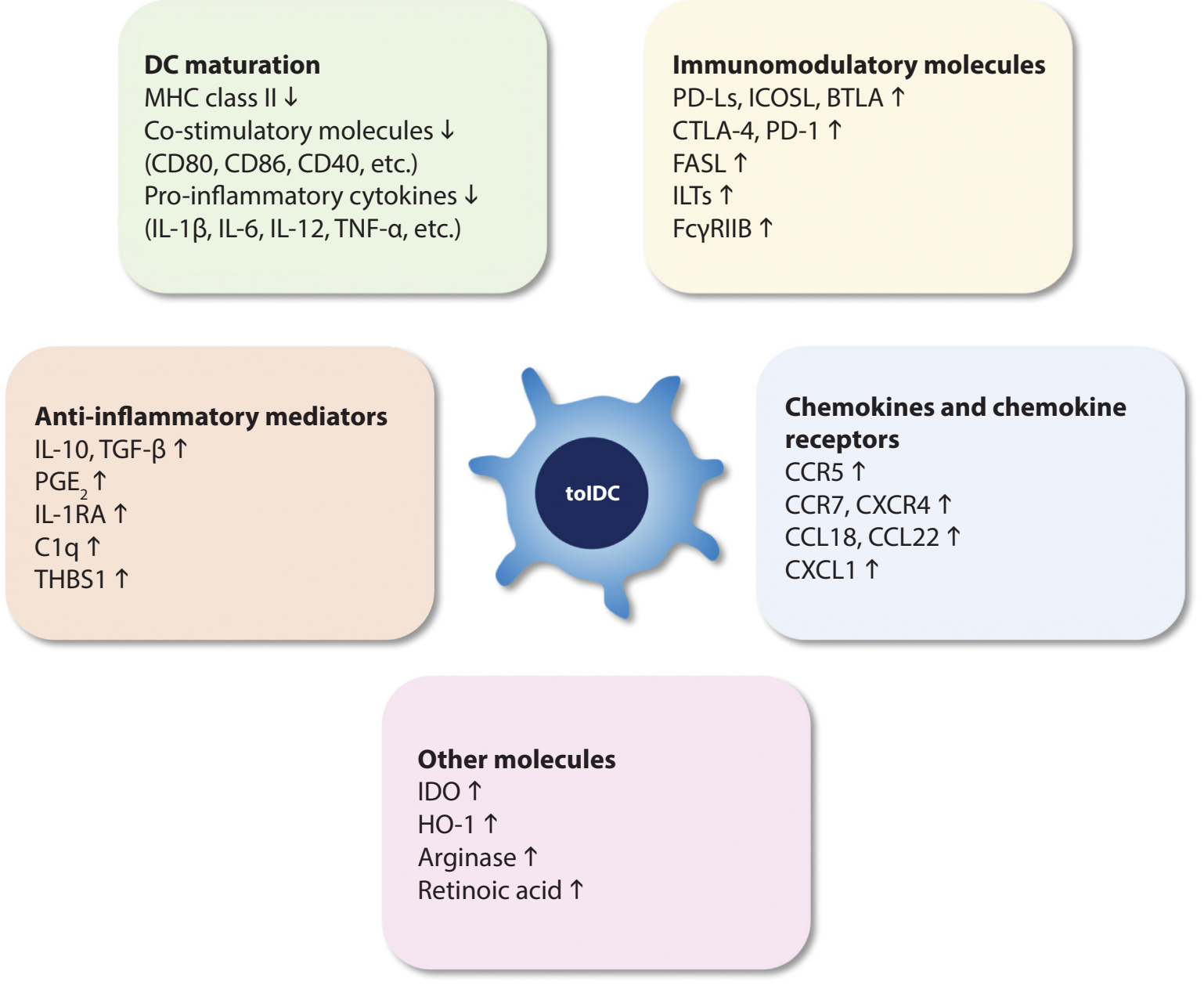

Figure 2. Phenotypic and functional features required for tol-DCs.

The key success of utilizing tol-DCs in autoimmune disease therapy is the functional quality of tol-DCs that is adequate for suppression of autoimmunity as well as induction of immune tolerance. Tolerogenicity of DCs is determined by the phenotypes, functions, and maturation status. Downmodulation of DC maturation, upregulation of immunomodulatory molecules, and increased production of anti-inflammatory mediators are important for negatively regulating $\mathrm{T}$ cell immunity, and supporting Treg induction and expansion. Expression of chemokines and chemokine receptors are essential for tolDCs migration and localization to lymphoid tissues and periphery to interact with T cells. Besides, several intrinsic molecules, such as IDO, HO-1, arginase, and retinoic acid, play key roles in modulating tolerogenicity of DCs. PD-Ls, programmed death-ligands; ICOSL, inducible T-cell co-stimulator ligand; BTLA, B and T lymphocyte attenuator; CTLA-4, cytotoxic T-lymphocyte-associated protein 4; PD-1, programmed death-1; FASL, Fas ligand; ILTs, inhibitory receptors Ig-like transcripts; PGE, prostaglandin E2; IL-1RA, interleukin-1 receptor antagonist; C1q, complement 1q; THBS1, thrombospondin-1; IDO, indoleamine 2,3-dioxygenase; HO-1, heme oxygenase- 1 .

Depletion of $\mathrm{pDC}$ and suppression of $\mathrm{pDC}$ functions are the alternative approaches to downmodulate type I IFN-mediated exacerbated inflammation. Plasmacytoid DC-specific marker BCDA2 is a receptor playing a role in antigen internalization and presentation. ${ }^{60}$ The in vitro treatment of pDCs obtained from SLE patients with anti-BDCA2 antibodies (BIIB059) could suppress pDC function and type I IFN production. ${ }^{61}$ Clinical trials in patients with SLE demonstrated that infusion of anti-BDCA2 inhibited type I IFN gene expression in the blood, diminished immune infiltration in the inflamed skin, and ameliorated cutaneous lupus lesions. ${ }^{62}$ Activation of TLR7 and TLR9 mediates signal transduction via myeloid differentiation primary response 88 (MyD88) and interleukin-1 receptor-associated kinase 4 (IRAK4), leading to type I IFN production, therefore, IRAK4 has become a potential therapeutic target in SLE. IRAK4 inhibitor could interfered with immune complex-induced type I IFN production in pDCs obtained from SLE patients. Furthermore, IRAK4 inhibitor suppressed many genes related to immune activation in those of pDCs. ${ }^{63}$ CD123 (IL-3Ra) is highly expressed on pDCs, and activation of CD123 is required for prolong survival of pDCs. ${ }^{64}$ Targeting pDCs by anti-CD123 (CSL362) led to the selective depletion of pDCs from peripheral blood cells of SLE patients via antibody-dependent cell-mediated cytotoxicity (ADCC) mechanism, and contributed to the reduced type I IFN and limited plasmablast expansion. ${ }^{65} \mathrm{Bcl}-2$ has been commonly known as a negative regulator for apoptotic cell death. Interestingly, Bcl-2 inhibitor 
A

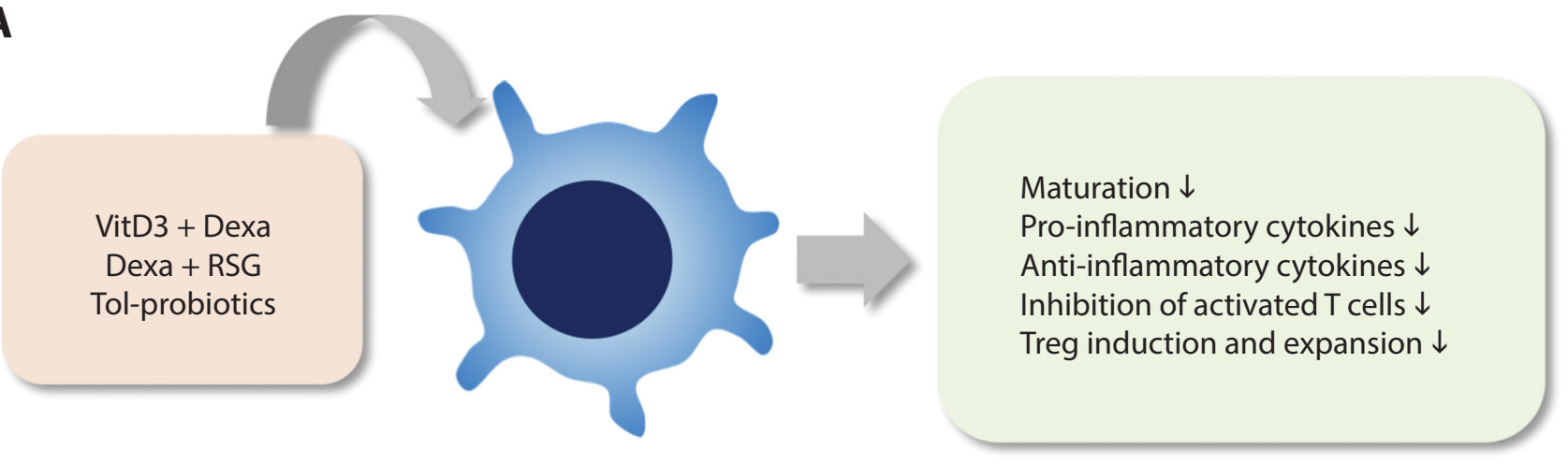

MoDCs from SLE patients

B

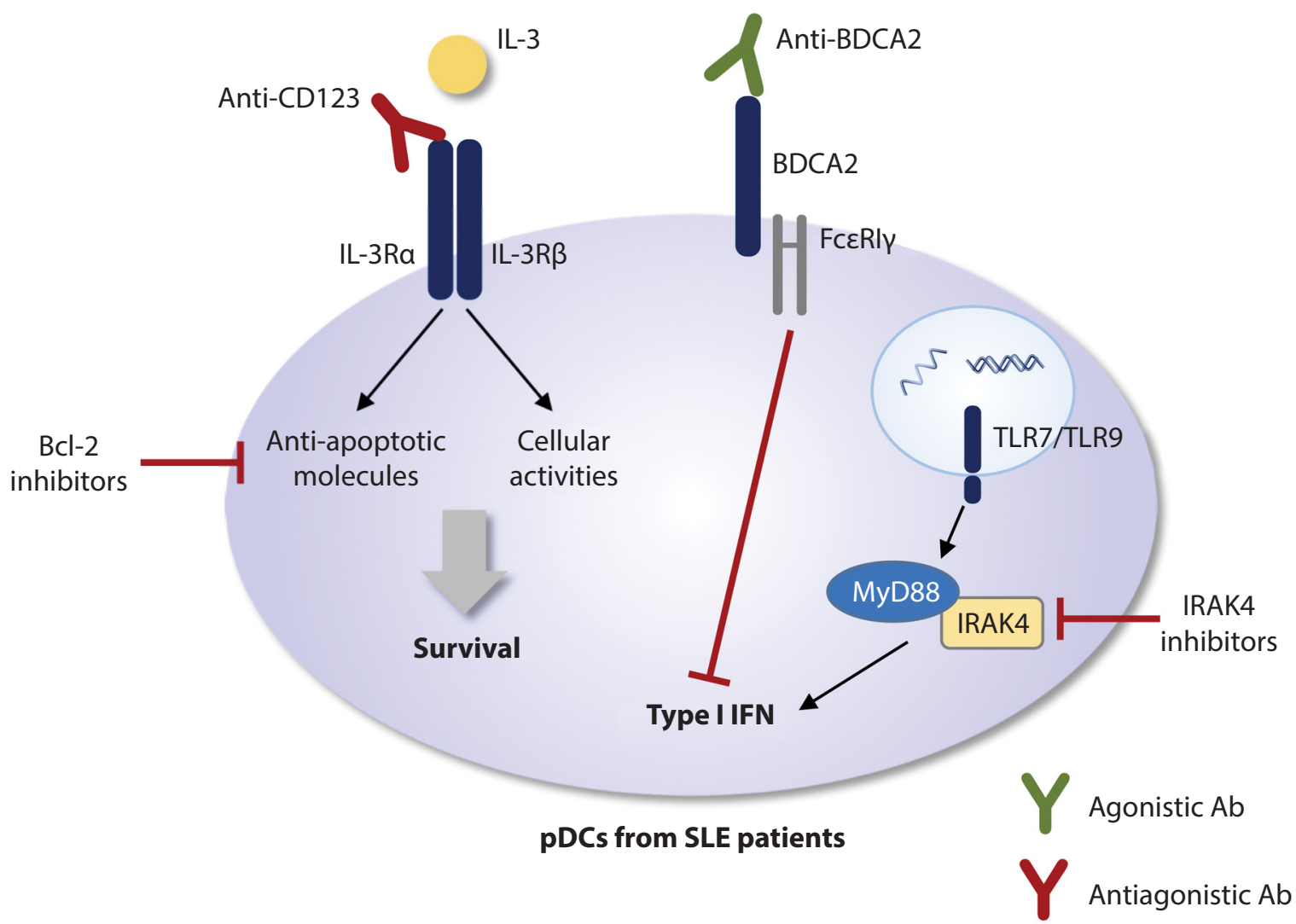

Figure 3. Summary of DC-targeted therapies in SLE.

The different strategies are utilized for targeting MoDCs and pDCs of SLE patients. A) Autologous MoDCs are skewed to tolerogenic phenotypes for the future infusion in SLE patients. B) Targeting pDCs emphasizes interfering with cell survival processes and inhibition of pDC activation and type I IFN production. VitD3, 1,25-dihydroxyvitamin D3; Dexa, dexmethazone; RSG, rosiglitazone; Tol-probiotics, tolerogenic probiotics.

selectively depleted pDCs in lupus prone mice and pDCs from peripheral blood of SLE patients, and it also inhibited type I IFN production. ${ }^{66}$ Female SLE patients medicated with a Bcl-2 inhibitor, venetoclax (ABT-199) showed the safety and efficacy, and the multiple ascending doses of venetoclax potentially killed autoreactive B cells. ${ }^{67}$

\section{Conclusion and perspectives}

One feasible key mechanism underlying SLE development and pathogenesis is alteration of DCs in number, phenotype, and function. In addition, interplays between DCs and adaptive immune components are critical for the disease amplification and perpetuation. Therefore, targeting DCs by interfering with their function or skewing them to tolerogenic phenotype will provide clinical advantages for SLE treatment (Figure 3). At present, there is limited information on clinical manipulation of DCs for SLE treatment which may be due to undetermined SLE-specific antigen and hidden clues in involvement of individual DC subsets in SLE pathogenesis. In addition, a wide variety of pharmaceutical and biological agents exploited for DC manipulation in $v i$ tro and in vivo produces the different phenotypic and functional features of DCs. Thorough understanding the role of DCs and their involvement in SLE pathogenesis will be thus 
beneficial for selecting the effective drugs that produce desirable properties of DCs, and allow us to reach the achievement of SLE therapy.

\section{Conflict of Interests}

The authors declare no conflict of interest.

\section{Acknowledgements}

This work was supported by funding from the International Network for Lupus Research (IRN59W0004) and Ratchadapisek Sompoch Endowment Fund, Chulalongkorn University (Health Cluster, 760001-HR). C. K. was supported by Ratchadapisek Sompoch fund for postdoctoral fellowship, Chulalongkorn University. Research Unit in Integrative Immuno-Microbial Biochemistry and Bioresponsive Nanomaterials and Center of Excellence in Immunology and Immune-mediated Diseases were supported by Ratchadapisek Sompoch Endowment Fund, Chulalongkorn University.

\section{References}

1. Durai V, Murphy KM. Functions of Murine Dendritic Cells. Immunity. 2016;45(4):719-36.

2. Collin M, Bigley V. Human dendritic cell subsets: an update. Immunology. 2018;154(1):3-20.

3. Doebel T, Voisin B, Nagao K. Langerhans Cells - The Macrophage in Dendritic Cell Clothing. Trends Immunol. 2017;38(11):817-28.

4. Clayton K, Vallejo AF, Davies J, Sirvent S, Polak ME. Langerhans Cells-Programmed by the Epidermis. Front Immunol. 2017;8:1676.

5. Maldonado-Lopez R, De Smedt T, Michel P, Godfroid J, Pajak B, Heirman C, et al. CD8alpha+ and CD8alpha- subclasses of dendritic cells direct the development of distinct T helper cells in vivo. J Exp Med. 1999;189(3):587-92.

6. den Haan JM, Lehar SM, Bevan MJ. CD8(+) but not CD8(-) dendritic cells cross-prime cytotoxic T cells in vivo. J Exp Med. 2000;192(12): 1685-96.

7. Jones A, Bourque J, Kuehm L, Opejin A, Teague RM, Gross C, et al. Immunomodulatory Functions of BTLA and HVEM Govern Induction of Extrathymic Regulatory T Cells and Tolerance by Dendritic Cells. Immunity. 2016;45(5):1066-77.

8. Coombes JL, Siddiqui KR, Arancibia-Carcamo CV, Hall J, Sun CM, Belkaid Y, et al. A functionally specialized population of mucosal CD103+ DCs induces Foxp3+ regulatory T cells via a TGF-beta and retinoic acid-dependent mechanism. J Exp Med. 2007;204(8):1757-64.

9. Fallarino F, Vacca C, Orabona C, Belladonna ML, Bianchi R, Marshall B, et al. Functional expression of indoleamine 2,3-dioxygenase by murine CD8 alpha(+) dendritic cells. Int Immunol. 2002;14(1):65-8.

10. Matteoli G, Mazzini E, Iliev ID, Mileti E, Fallarino F, Puccetti P, et al. Gut CD103+ dendritic cells express indoleamine 2,3-dioxygenase which influences $\mathrm{T}$ regulatory/ $\mathrm{T}$ effector cell balance and oral tolerance induction. Gut. 2010;59(5):595-604.

11. Persson EK, Uronen-Hansson H, Semmrich M, Rivollier A, Hagerbrand $\mathrm{K}$, Marsal J, et al. IRF4 transcription-factor-dependent CD103(+) $\mathrm{CD} 11 \mathrm{~b}(+)$ dendritic cells drive mucosal T helper 17 cell differentiation. Immunity. 2013;38(5):958-69.

12. Gao Y, Nish SA, Jiang R, Hou L, Licona-Limon P, Weinstein JS, et al. Control of $\mathrm{T}$ helper 2 responses by transcription factor IRF4-dependent dendritic cells. Immunity. 2013;39(4):722-32.

13. Briseno CG, Satpathy AT, Davidson JTt, Ferris ST, Durai V, Bagadia P, et al. Notch2-dependent DC2s mediate splenic germinal center responses. Proc Natl Acad Sci U S A. 2018;115(42):10726-31.

14. Proietto AI, van Dommelen S, Zhou P, Rizzitelli A, D'Amico A, Steptoe RJ, et al. Dendritic cells in the thymus contribute to T-regulatory cell induction. Proc Natl Acad Sci U S A. 2008;105(50):19869-74.

15. Guilliams M, Crozat K, Henri S, Tamoutounour S, Grenot P, Devilard E, et al. Skin-draining lymph nodes contain dermis-derived CD103(-) dendritic cells that constitutively produce retinoic acid and induce Foxp3 (+) regulatory T cells. Blood. 2010;115(10):1958-68.
16. Belladonna ML, Grohmann U, Guidetti P, Volpi C, Bianchi R, Fioretti $\mathrm{MC}$, et al. Kynurenine pathway enzymes in dendritic cells initiate tolerogenesis in the absence of functional IDO. J Immunol. 2006;177(1): $130-7$.

17. Yun TJ, Lee JS, Machmach K, Shim D, Choi J, Wi YJ, et al. Indoleamine 2,3-Dioxygenase-Expressing Aortic Plasmacytoid Dendritic Cells Protect against Atherosclerosis by Induction of Regulatory T Cells. Cell Metab. 2016;23(5):852-66.

18. Mittag D, Proietto AI, Loudovaris T, Mannering SI, Vremec D, Shortman $\mathrm{K}$, et al. Human dendritic cell subsets from spleen and blood are similar in phenotype and function but modified by donor health status. J Immunol. 2011;186(11):6207-17.

19. Wang $\mathrm{YH}$, Ito T, Wang $\mathrm{YH}$, Homey B, Watanabe $\mathrm{N}$, Martin R, et al. Maintenance and polarization of human $\mathrm{TH} 2$ central memory $\mathrm{T}$ cells by thymic stromal lymphopoietin-activated dendritic cells. Immunity. 2006;24(6):827-38.

20. Comi M, Avancini D, Santoni de Sio F, Villa M, Uyeda MJ, Floris M, et al. Coexpression of CD163 and CD141 identifies human circulating IL-10-producing dendritic cells (DC-10). Cell Mol Immunol. 2019.

21. Tsoumakidou M, Tousa S, Semitekolou M, Panagiotou P, Panagiotou A, Morianos I, et al. Tolerogenic signaling by pulmonary CD1c+ dendritic cells induces regulatory $\mathrm{T}$ cells in patients with chronic obstructive pulmonary disease by IL-27/IL-10/inducible costimulator ligand. J Allergy Clin Immunol. 2014;134(4):944-54 e8.

22. Jahrsdorfer B, Vollmer A, Blackwell SE, Maier J, Sontheimer K, Beyer $\mathrm{T}$, et al. Granzyme B produced by human plasmacytoid dendritic cells suppresses T-cell expansion. Blood. 2010;115(6):1156-65.

23. Conrad C, Gregorio J, Wang YH, Ito T, Meller S, Hanabuchi S, et al. Plasmacytoid dendritic cells promote immunosuppression in ovarian cancer via ICOS costimulation of Foxp3(+) T-regulatory cells. Cancer Res. 2012;72(20):5240-9.

24. Fiore N, Castellano G, Blasi A, Capobianco C, Loverre A, Montinaro $\mathrm{V}$, et al. Immature myeloid and plasmacytoid dendritic cells infiltrate renal tubulointerstitium in patients with lupus nephritis. Mol Immunol. 2008;45(1):259-65.

25. Blomberg S, Eloranta ML, Cederblad B, Nordlin K, Alm GV, Ronnblom L. Presence of cutaneous interferon-alpha producing cells in patients with systemic lupus erythematosus. Lupus. 2001;10(7):484-90.

26. Sahu R, Bethunaickan R, Singh S, Davidson A. Structure and function of renal macrophages and dendritic cells from lupus-prone mice. Arthritis Rheumatol. 2014;66(6):1596-607.

27. Zhou Z, Ma J, Xiao C, Han X, Qiu R, Wang Y, et al. Phenotypic and functional alterations of pDCs in lupus-prone mice. Sci Rep. 2016;6: 20373.

28. Shao WH, Cohen PL. Disturbances of apoptotic cell clearance in systemic lupus erythematosus. Arthritis Res Ther. 2011;13(1):202.

29. Santiago-Raber ML, Baudino L, Izui S. Emerging roles of TLR7 and TLR9 in murine SLE. J Autoimmun. 2009;33(3-4):231-8.

30. Hardy MP, Audemard E, Migneault F, Feghaly A, Brochu S, Gendron P, et al. Apoptotic endothelial cells release small extracellular vesicles loaded with immunostimulatory viral-like RNAs. Sci Rep. 2019;9(1):7203.

31. Means TK, Latz E, Hayashi F, Murali MR, Golenbock DT, Luster AD. Human lupus autoantibody-DNA complexes activate DCs through cooperation of CD32 and TLR9. J Clin Invest. 2005;115(2):407-17.

32. Santiago-Raber ML, Baccala R, Haraldsson KM, Choubey D, Stewart TA, Kono $\mathrm{DH}$, et al. Type-I interferon receptor deficiency reduces lupus-like disease in NZB mice. J Exp Med. 2003;197(6):777-88.

33. Davison LM, Jorgensen TN. Sialic acid-binding immunoglobulin-type lectin H-positive plasmacytoid dendritic cells drive spontaneous lupus-like disease development in B6.Nba2 mice. Arthritis Rheumatol. 2015;67(4):1012-22.

34. Mauri C, Menon M. The many faces of type I interferon in systemic lupus erythematosus. J Clin Invest. 2015;125(7):2562-4.

35. Blanco P, Palucka AK, Gill M, Pascual V, Banchereau J. Induction of dendritic cell differentiation by IFN-alpha in systemic lupus erythematosus. Science. 2001;294(5546):1540-3.

36. Lopez P, Rodriguez-Carrio J, Caminal-Montero L, Mozo L, Suarez A. A pathogenic IFNalpha, BLyS and IL-17 axis in Systemic Lupus Erythematosus patients. Sci Rep. 2016;6:20651.

37. Yu CF, Peng WM, Oldenburg J, Hoch J, Bieber T, Limmer A, et al. Human plasmacytoid dendritic cells support Th17 cell effector function in response to TLR7 ligation. J Immunol. 2010;184(3):1159-67. 
38. Venereau E, Ceriotti C, Bianchi ME. DAMPs from Cell Death to New Life. Front Immunol. 2015;6:422.

39. Tanaka A, Ito $T$, Kibata $K$, Inagaki-Katashiba $N$, Amuro $H$, Nishizawa T, et al. Serum high-mobility group box 1 is correlated with interferon -alpha and may predict disease activity in patients with systemic lupus erythematosus. Lupus. 2019;28(9):1120-7.

40. Tian J, Avalos AM, Mao SY, Chen B, Senthil K, Wu H, et al. Toll-like receptor 9-dependent activation by DNA-containing immune complexes is mediated by HMGB1 and RAGE. Nat Immunol. 2007;8(5):487-96.

41. Papp K, Vegh P, Hobor R, Szittner Z, Voko Z, Podani J, et al. Immune complex signatures of patients with active and inactive SLE revealed by multiplex protein binding analysis on antigen microarrays. PLoS One. 2012;7(9):e44824.

42. Barrat FJ, Meeker T, Gregorio J, Chan JH, Uematsu S, Akira S, et al. Nucleic acids of mammalian origin can act as endogenous ligands for Toll-like receptors and may promote systemic lupus erythematosus. J Exp Med. 2005;202(8):1131-9.

43. Leonard D, Eloranta ML, Hagberg N, Berggren O, Tandre K, Alm $\mathrm{G}$, et al. Activated $\mathrm{T}$ cells enhance interferon-alpha production by plasmacytoid dendritic cells stimulated with RNA-containing immune complexes. Ann Rheum Dis. 2016;75(9):1728-34.

44. Fransen JH, Hilbrands LB, Jacobs CW, Adema GJ, Berden JH, Van der Vlag J. Both early and late apoptotic blebs are taken up by DC and induce IL-6 production. Autoimmunity. 2009;42(4):325-7.

45. Fransen JH, Hilbrands LB, Ruben J, Stoffels M, Adema GJ, van der Vlag J, et al. Mouse dendritic cells matured by ingestion of apoptotic blebs induce $\mathrm{T}$ cells to produce interleukin-17. Arthritis Rheum. 2009;60(8):2304-13

46. Deng GM, Liu L, Kyttaris VC, Tsokos GC. Lupus serum IgG induces skin inflammation through the TNFR1 signaling pathway. J Immunol. 2010;184(12):7154-61

47. Heesters BA, Chatterjee P, Kim YA, Gonzalez SF, Kuligowski MP, Kirchhausen T, et al. Endocytosis and recycling of immune complexes by follicular dendritic cells enhances B cell antigen binding and activation. Immunity. 2013;38(6):1164-75.

48. Sauma D, Crisostomo N, Fuentes C, Gleisner MA, Hidalgo Y, Fuenzalida MJ, et al. Adoptive transfer of autoimmune splenic dendritic cells to lupus-prone mice triggers a B lymphocyte humoral response. Immunol Res. 2017;65(4):957-68.

49. Ols ML, Cullen JL, Turqueti-Neves A, Giles J, Shlomchik MJ. Dendritic Cells Regulate Extrafollicular Autoreactive B Cells via T Cells Expressing Fas and Fas Ligand. Immunity. 2016;45(5):1052-65.

50. Decker P, Kotter I, Klein R, Berner B, Rammensee HG. Monocyte -derived dendritic cells over-express CD86 in patients with systemic lupus erythematosus. Rheumatology (Oxford). 2006;45(9):1087-95.

51. Jensen MA, Patterson KC, Kumar AA, Kumabe M, Franek BS, Niewold TB. Functional genetic polymorphisms in ILT3 are associated with decreased surface expression on dendritic cells and increased serum cytokines in lupus patients. Ann Rheum Dis. 2013;72(4):596-601.

52. Guerra-de Blas Pdel C, Villasenor-Talavera YS, Cruz-Gonzalez Dde J, Baranda L, Doniz-Padilla L, Abud-Mendoza C, et al. Analysis of the Expression and Function of Immunoglobulin-Like Transcript 4 (ILT4, LILRB2) in Dendritic Cells from Patients with Systemic Lupus Erythematosus. J Immunol Res. 2016;2016:4163094.

53. Kanakoudi-Tsakalidou F, Farmaki E, Tzimouli V, Taparkou A, Paterakis G, Trachana M, et al. Simultaneous changes in serum HMGB1 and IFN-alpha levels and in LAIR-1 expression on plasmatoid dendritic cells of patients with juvenile SLE. New therapeutic options? Lupus. 2014; 23(3):305-12
54. Obregon C, Kumar R, Pascual MA, Vassalli G, Golshayan D. Update on Dendritic Cell-Induced Immunological and Clinical Tolerance. Front Immunol. 2017;8:1514.

55. Navarro-Barriuso J, Mansilla MJ, Martinez-Caceres EM. Searching for the Transcriptomic Signature of Immune Tolerance Induction -Biomarkers of Safety and Functionality for Tolerogenic Dendritic Cells and Regulatory Macrophages. Front Immunol. 2018;9:2062.

56. Berkun Y, Verbovetski I, Ben-Ami A, Paran D, Caspi D, Krispin A et al. Altered dendritic cells with tolerizing phenotype in patients with systemic lupus erythematosus. Eur J Immunol. 2008;38(10):2896-904.

57. Wu HJ, Lo Y, Luk D, Lau CS, Lu L, Mok MY. Alternatively activated dendritic cells derived from systemic lupus erythematosus patients have tolerogenic phenotype and function. Clinical immunology. 2015; 156(1):43-57.

58. Obreque J, Vega F, Torres A, Cuitino L, Mackern-Oberti JP, Viviani P, et al. Autologous tolerogenic dendritic cells derived from monocytes of systemic lupus erythematosus patients and healthy donors show a stable and immunosuppressive phenotype. Immunology. 2017;152(4):648-59.

59. Esmaeili SA, Mahmoudi M, Rezaieyazdi Z, Sahebari M, Tabasi N, Sahebkar A, et al. Generation of tolerogenic dendritic cells using Lactobacillus rhamnosus and Lactobacillus delbrueckii as tolerogenic probiotics. J Cell Biochem. 2018;119(9):7865-72.

60. Dzionek A, Sohma Y, Nagafune J, Cella M, Colonna M, Facchetti F, et al. BDCA-2, a novel plasmacytoid dendritic cell-specific type II C-type lectin, mediates antigen capture and is a potent inhibitor of interferon alpha/beta induction. J Exp Med. 2001;194(12):1823-34.

61. Pellerin A, Otero K, Czerkowicz JM, Kerns HM, Shapiro RI, Ranger AM, et al. Anti-BDCA2 monoclonal antibody inhibits plasmacytoid dendritic cell activation through $\mathrm{Fc}$-dependent and Fc-independent mechanisms. EMBO Mol Med. 2015;7(4):464-76.

62. Furie R, Werth VP, Merola JF, Stevenson L, Reynolds TL, Naik H, et al. Monoclonal antibody targeting BDCA2 ameliorates skin lesions in systemic lupus erythematosus. J Clin Invest. 2019;129(3):1359-71.

63. Hjorton K, Hagberg N, Israelsson E, Jinton L, Berggren O, Sandling JK, et al. Cytokine production by activated plasmacytoid dendritic cells and natural killer cells is suppressed by an IRAK4 inhibitor. Arthritis Res Ther. 2018;20(1):238

64. Ghirelli C, Zollinger R, Soumelis V. Systematic cytokine receptor profiling reveals GM-CSF as a novel TLR-independent activator of human plasmacytoid predendritic cells. Blood. 2010;115(24):5037-40.

65. Oon S, Huynh H, Tai TY, Ng M, Monaghan K, Biondo M, et al. A cytotoxic anti-IL-3Ralpha antibody targets key cells and cytokines implicated in systemic lupus erythematosus. JCI Insight. 2016;1(6): e86131.

66. Zhan Y, Carrington EM, Ko HJ, Vikstrom IB, Oon S, Zhang JG, et al. Bcl-2 antagonists kill plasmacytoid dendritic cells from lupus-prone mice and dampen interferon-alpha production. Arthritis Rheumatol. 2015;67(3):797-808.

67. Lu P, Fleischmann R, Curtis C, Ignatenko S, Clarke SH, Desai M, et al. Safety and pharmacodynamics of venetoclax (ABT-199) in a randomized single and multiple ascending dose study in women with systemic lupus erythematosus. Lupus. 2018;27(2):290-302. 